\title{
Simultaneous en-face imaging of two layers in the human retina by low-coherence reflectometry
}

\author{
Adrian Gh. Podoleanu, George M. Dobre, David J. Webb, and David A. Jackson \\ Applied Optics Group, Physics Laboratory, University of Kent, Canterbury, CT2 7NR, UK \\ Received January 28, 1997 \\ We show that with a fiberized multiple Michelson-interferometer-type configuration, transverse images from \\ several layers in the human eye can be simultaneously obtained. We demonstrate the principle by producing \\ simultaneous $100 \times 100$ pixel en-face images of a $4 \mathrm{~mm} \times 4 \mathrm{~mm}$ region on a postmortem retina for two depth \\ positions $250 \mu \mathrm{m}$ apart. (C) 1997 Optical Society of America
}

Low-coherence reflectometry (LCR) has emerged as a method for absolute surface profile and distance determination with very good axial and transverse accuracy and has been applied to reflectance measurements of different structures. ${ }^{1,2}$ LCR was also successfully applied to in vitro and in vivo imaging of different biological tissues. ${ }^{3,4}$ Lately a great deal of attention has been devoted to investigations of the eye and retinal imaging. ${ }^{4}$

LCR has the capability of achieving very good depth accuracies (down to $3.7 \mu \mathrm{m}$, as demonstrated with a Kerr-lens mode-locked Ti: $\mathrm{Al}_{2} \mathrm{O}_{3}$ laser $^{3}$ ). This means that LCR can be used to perform optical biopsies and is a powerful method for noninvasive optical diagnostics in vivo. However, single en-face images are somewhat difficult to interpret because they show only fragments of anatomical structures owing to such a high depth discrimination. Therefore simultaneously displaying multiple images at different depths would be useful and could also lead to building a three-dimensional profile of the object in real time.

In this Letter we discuss and demonstrate simultaneous production of LCR images by using a configuration of multiple single-mode fiber interferometers. Configurations of two bulk interferometers ${ }^{5,6}$ for eye investigations have been reported in which the interferometers were used in tandem to eliminate axial eye movements. Our proposal is different, as we aim to have the potential to use a large number of independent interferometers.

We illustrate the method by acquiring and simultaneously displaying two en-face images from the retina of a postmortem human eye. The setup is shown in Fig. 1. Light from a pigtailed superluminescent diode (SLD; Superlum SLD-361) is injected into a singlemode directional coupler, DC2. The transmitted light from one of the output ports is directed to mirror M2, and light from the other port is injected into a second single-mode coupler, DC1, whose outputs provide a reference beam entering collimator $\mathrm{C} 1$ and a target beam entering a microscope objective C3. Two Michelson interferometers are formed by using this arrangement. The reference arm in each interferometer consists of a microscope objective, $\mathrm{C} 1$ (C2) and a mirror, M1 (M2). Both mirrors are mounted upon the same computer-controlled translation stage, TS. The fiber ends before $\mathrm{C} 1, \mathrm{C} 2$, and $\mathrm{C} 3$ are polished at a $10^{\circ}$ angle to reduce the noise associated with backreflected light.
One can adjust the optical path difference (OPD) between the two interferometers (chosen in our case to be $250 \mu \mathrm{m}$ ) by shifting the supports of the fiber ends and collimator $\mathrm{C} 1$ (C2) relative to mirror M1 (M2), which are vibrated by two electrostrictive elements, EE's, driven by sinusoidal generators G1 and G2, at $f_{1}=30 \mathrm{kHz}$ and $f_{2}=22.5 \mathrm{kHz}$, respectively. We adjusted the amplitude of the driving signals to maximize the modulation at $2 f_{1}$ and $2 f_{2}$ (the argument of the second-order Bessel function of the first kind is $\approx 3$ ). After collimation, the beam in the sensing arm enters an orthogonal scanning mirror pair SXY and passes through a converging lens of $60-\mathrm{mm}$ focal length L1 before entering a model eye ME consisting of a 1-cm-diameter convergent lens of focal length $30 \mathrm{~mm}, \mathrm{~L} 2$, and a container with a human retina in formalin, HR. The retinal tissue is placed in the focal plane of lens L2. The beam spot on the last mirror in SXY and the anterior focus of L2 are conjugate points by virtue of L1. Two photodetectors, PD1 and PD2, collect the returned optical signals. After photodetection, the signals are bandpass filtered BPF at $2 f_{1}\left(2 f_{2}\right)$ (to avoid the residual intensity modulation at $f$ ), then rectified $\mathrm{R}$ and low-pass filtered LPF. The triangle function generator, TX, drives the horizontal line scanner, MX, and triggers, by an analog-digital interface, the acquisition of the two signals from LPF outputs. Data acquisition and hardware commands are synchronized under the control of a LabView Virtual Instrument, which also produces incremental voltage steps by digital-analog interface to drive the vertical scanner, MY.

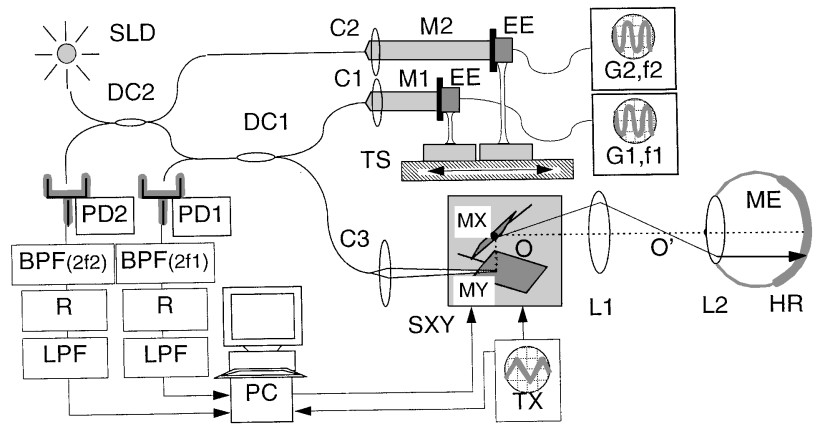

Fig. 1. Experimental arrangement. See text for definitions. 
With MX driven at a frequency $F=20 \mathrm{~Hz}$ and a peak-peak amplitude $1 \mathrm{~V}$, and with MY driven more than 100 steps from -0.5 to $0.5 \mathrm{~V}$, the images in Fig. 2 were obtained. For both mirrors an electrical bias of $1 \mathrm{~V}$ produces a $4^{\circ}$ deflection. A scan area of $4 \mathrm{~mm} \times 4 \mathrm{~mm}$ is thus generated upon the target. Notice that the lower image for $z=z^{\prime}$ becomes similar to the upper one at $z=z^{\prime}+250 \mu \mathrm{m}$, i.e., the OPD's in the two interferometers are separated by $250 \mu \mathrm{m}$. Both images in each pair shown in Fig. 2 were simultaneously acquired and displayed in less than $3 \mathrm{~s}$.

The production of two images is not done at the expense of speed or signal-noise ratio in the first interferometer. The addition of a second coupler (and interferometer) is compensated for by a corresponding increase in optical power, so there is no penalty for adding a second interferometer (if one neglects the increase in Rayleigh scattering that is due to longer fiber lengths). However, the image quality obtained in the second interferometer may not be as good as in the first interferometer, as is shown below.

This method could be extended to display $n$ layers in depth by use of a setup similar to that shown in Fig. 1 with $n$ interferometers. If we consider the power from the SLD launched into the fiber to be $P_{0}$, the photodetectors to have sensitivity $\eta$, the directional couplers DC to have zero loss, the reflectivity of mirrors $\mathrm{M}_{j}$ to be $R_{j}$, and the eye to return a fraction $O$ of the incident power (both $R_{j}$ and $O$ are adjusted to include coupling losses in and out of the fiber), the photocurrent at the last photodetector, $\mathrm{PD} n$, is given by

$$
\begin{aligned}
i_{n}= & \eta P_{0}\left[\frac{O}{2^{2 n}}+\frac{1}{4} \sum_{j=1}^{n} \frac{R_{j}}{2^{2(n-j)}}\right. \\
& +\frac{(-1)^{n-1}}{2^{n}} \sqrt{O R_{n}} \gamma\left(d_{O n}\right) \cos \left(\frac{2 \pi}{\lambda} d_{O n}\right) \\
& +\sum_{j=1}^{n-1} c_{j}(n) \sqrt{O R_{j}} \gamma\left(d_{O j}\right) \cos \left(\frac{2 \pi}{\lambda} d_{O j}\right) \\
& \left.+\sum_{j, p=1}^{n} e_{j p}(n) \sqrt{R_{p} R_{j}} \gamma\left(\mathrm{d}_{p j}\right)\left(\cos \frac{2 \pi}{\lambda} d_{p j}\right)\right]
\end{aligned}
$$

where $\gamma$ stands for the modulus of the optical field correlation function, $d_{O j}$ is the OPD in interferometer $j$, $d_{p j}$ is the OPD between mirrors $\mathrm{M}_{j}$ and $\mathrm{M} p, c_{j}$ and $e_{j p}$ are numerical coefficients, and $\lambda$ is the central wavelength of the SLD. The first two terms represent the bias, and the third one is the useful signal, which is periodic with components at multiples of $f_{n}$. The fourth term represents interference events between the object and the previous reference mirrors, $j=1, \ldots, n-1$. These are periodic terms at multiples of frequencies $f_{j}$ with $j \neq n$, which can be sufficiently attenuated by the bandpass filter tuned to $f_{n}$ if the frequency values $f_{j}$ are correctly selected. The last term represents interference events between the signals reflected by the mirrors $\mathrm{M} j$ and $\mathrm{M} p$ with $p \neq j$. These become neglible when $d_{p j}$ are adjusted to exceed the coherence length $l_{c}$. In the configuration shown in Fig. 1, $d_{12}=250 \mu \mathrm{m}$, which is much longer than the $25-\mu \mathrm{m}$ coherence length of the source.

It can be shown that the object power decreases from one interferometer to the next and the reference power increases by the same factor. Consequently, a similar amplitude for the useful interference signal results at all photodetectors, given by the third term in Eq. (1).

The maximum number of layers is given by $N=$ $C P / l_{c}$, where $C P$ is the width of the confocal profile (depth of focus range). In our case, with $C P \approx 500 \mu \mathrm{m}$ and $l_{c}=25 \mu \mathrm{m}$, the value of $N$ is 20 .

Any increase in the number of layers should be accompanied by a corresponding increase in the optical source power. For safety reasons the power of the beam entering the living eye should be limited to a value that we call $P_{s}$, with $P_{0} / 2^{n}=P_{s}$ in Eq. (1). It can be clearly seen from Eq. (1) that the useful term remains constant as $n$ increases if the source power is correspondingly increased. This increase in power is feasible with existing large-bandwidth optical sources. The images in Fig. 2 were obtained with an SLD, which delivered $90-\mu \mathrm{W}$ power that was incident upon lens L2. As much as $P_{s}=0.2 \mathrm{~mW}$ cw power could be injected into the eye at $800 \mathrm{~nm} .{ }^{6}$ Given that the average power of solid-state large-bandwidth lasers ${ }^{3}$ such as a Kerr-lens mode-locked Ti: $\mathrm{Al}_{2} \mathrm{O}_{3}$ can exceed $400 \mathrm{~mW}$, up to 11 lossless interferometers can, in principle, operate simultaneously and still allow the delivery of $0.2-\mathrm{mW}$ power to the eye. However, as Eq. (1) shows, another limitation to the number of layers would be the saturation power at the level of the $n$th photodetector. If a photodetector saturation limit of $1 \mathrm{~mW}$ is imposed (for illustration only; higher values can be accommodated in practice), this would restrict the optical source power to $4 \mathrm{~mW}$. For $P_{s}=0.2 \mathrm{~mW}$ injected into the eye, no more than four interferometers could be used, as in this case $2^{4} \times 0.2=3.2 \mathrm{~mW}$ and $2^{5} \times 0.2=6.4>4 \mathrm{~mW}$. The diagram in Fig. 3 shows the concept extended to four interferometers.

Another important constraint on increasing the number of layers (and accordingly of interferometers) derives from the fact that the interference signal from the first interferometer is present on the photodetector in the second interferometer, the interference signal from the second is present in the third interferometer,

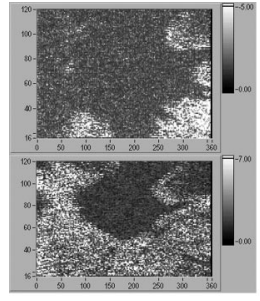

000

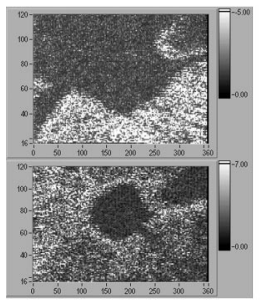

150

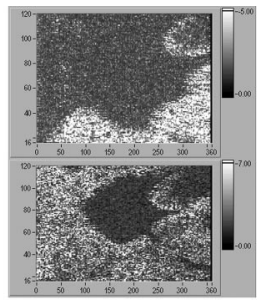

050

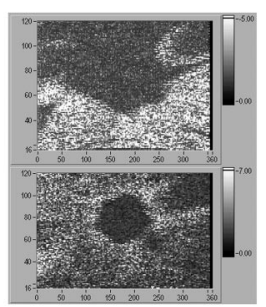

200

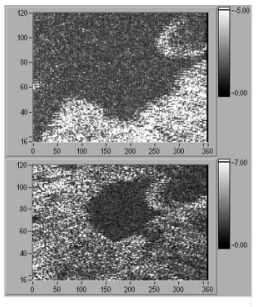

100

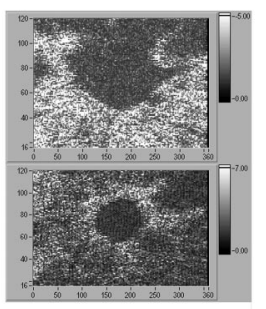

250
Fig. 2. Six pairs of $4 \mathrm{~mm} \times 4 \mathrm{~mm}$ en-face images taken at $50-\mu \mathrm{m}$ intervals. 


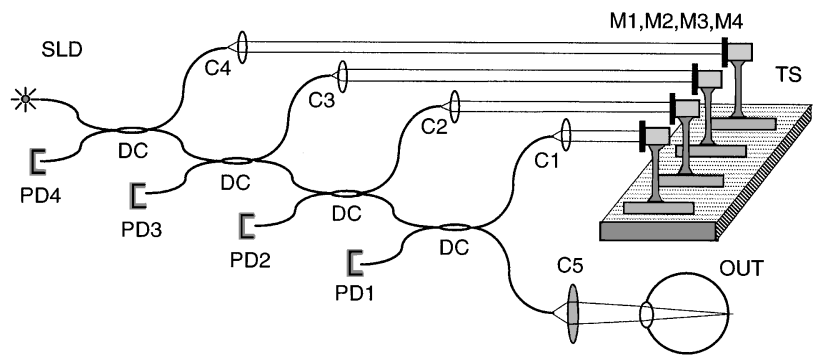

Fig. 3. System with four couplers (interferometers) for the simultaneous display of four layers: SLD, low-coherence pigtailed optical source (superluminescent diode); DC's, 50:50 single-mode directional couplers; PD1-PD4, photodetectors; M1-M4, mirrors; TS, computer-controllable translation stage; C1-C5, microscope objectives; OUT, object under test.

and so on. The electronics in the $n$th interferometer should filter out $n-1$ interference signals, as shown by the fourth term in Eq. (1). This cross-talk noise can be reduced by a correct selection of frequencies $f_{j}$. Our electrostrictive elements can vibrate millimetersize mirrors by as much as $35 \mathrm{kHz}$ with amplitudes well above one wavelength. The electrical bandwidth $2 B$ about each carrier $f_{j}$ can be approximately evaluated as $2 B \approx 2 M F$, where the number of pixels $M$ is given by $M=w / d$, with $w$ the linewidth (image width on the target) and $d$ the diffraction-limited spot size. In our case the minimum spot size is $d=40 \mu \mathrm{m}$ (close to the value achievable with an undilated eye ${ }^{6}$ ). If we use $w=4 \mathrm{~mm}$ (i.e., $M=100$ ) and $F=20 \mathrm{~Hz}$, then $2 B \approx 4 \mathrm{kHz}$. We allow for windows of $2 B$ bandwidth about $2 f_{1}, 2 f_{2}, 2 f_{3}, 2 f_{4}$, and $3 f_{1}$ (considering $f_{1}$ the smallest of the set $f_{1}, \ldots f_{4}$ ), with a clearance of at least $B$ between adjacent windows, we use a possible set of frequencies: $f_{1}=24, f_{2}=27, f_{3}=30$, and $f_{4}=33 \mathrm{kHz}$. Higher modulation frequencies could be achieved by electro-optic crystals, in which case another, unmodulated crystal should be placed in the sensing arm to compensate for dispersion. ${ }^{3}$

In addition, as Eq. (1) shows, the bias increases with $n$. The associated contribution to noise of the constant power of the detector [represented mainly by the second term in Eq. (1)] could be kept low only by reduction of the bandwidth, i.e., an increase in the image-acquisition time. Considering that all bandpass filters have the same bandwidth, and that all the unwanted interference signals [the fourth term in Eq. (1)] are largely attenuated, and assuming that $O \ll R_{j} \cong 1$, the only noise contribution comes from the second term in Eq. (1), denoted $I_{\text {ref }}$ (due to optical power returned from the reference arms). We can now evaluate the signal-noise ratio in each interferometer by substituting this photocurrent into the mean-square current fluctuation given by ${ }^{7}$

$$
\left\langle\Delta I_{n}{ }^{2}\right\rangle=2 e B\left\langle I_{\text {ref }}\right\rangle+B\left(\left\langle I_{\text {ref }}\right\rangle / \Delta \nu\right),
$$

where $\Delta \nu$ is the optical linewidth (12 THz for our SLD) and $e$ is the electron charge. For the interference signal to prevail over thermal and electronic noise, it can be shown that a minimum optical power is needed, making the second term in Eq. (2) dominant (excessphoton-noise limited). In this case, compared with that of the first interferometer, the signal-noise ratio decreases by $\sim 8 \mathrm{~dB}$ in the second interferometer, by $14 \mathrm{~dB}$ in the third interferometer and by $21 \mathrm{~dB}$ in the fourth interferometer.

We have discussed a simple configuration. A morecomplex configuration can be designed that uses an extra coupler in each interferometer and replaces the flat mirrors with corner-cube mirrors. In this way a balanced photodetection technique ${ }^{8}$ can be implemented, with the advantage that the first term in an equation similar to Eq. (2) for balanced detection prevails (shot-noise-limited regime), and consequently superior signal-noise ratios are obtained. In addition, the interferometers are independent of each other.

In conclusion, a LCR system capable of producing two simultaneous en-face images at two different depths in the human retina in vitro has been demonstrated. The same setup is equally capable of producing two simultaneous logitudinal images through a combination of one transverse scan ( $x$ or $y$ ) and a scan of the translation stage in $z$ at constant speed.

\section{References}

1. R. C. Youngquist, S. Carr, and D. E. N. Davies, Opt. Lett. 12, 158 (1987).

2. A. G. Podoleanu, G. M. Dobre, D. J. Webb, and D. A. Jackson, Opt. Lett. 21, 1789 (1996).

3. B. E. Bouma, G. J. Tearney, S. A. Boppart, M. R. Hee, M. E. Brezinski, and J. G. Fujimoto, Opt. Lett. 20, 1486 (1995).

4. C. A. Puliafito, M. R. Hee, C. P. Lin, E. Reichel, J. S. Schuman, J. S. Duker, J. A. Izatt, E. A. Swanson, and J. G. Fujimoto, Opthalmology 102, 217 (1995).

5. A. F. Fercher, J. Biomed. Opt. 1, 157 (1996).

6. W. Drexler, C. K. Hitzenberger, H. Sattmann, and A. F. Fercher, Opt. Eng. 34, 701 (1995).

7. P. R. Morkel, R. I. Laming, and D. N. Payne, Electron. Lett. 26, 96 (1990).

8. K. Takada, A. Himeno, and K. Yukimatsu, Appl. Phys. Lett. 59, 2483 (1991). 